\title{
Diseño de una bebida potencialmente funcional de tomate (Lycopersicum esculentum) con tumeric (Curcuma longa Linn)
}

\section{(Design of a functional beverage of tomato juice (Lycopersicon esculentum) with tumeric (Curcuma longa Linn))}

*Nubia Lisbeth Matute Castro ${ }^{1}$, Gloria Panades Ambrosio ${ }^{2}$, Luis Cruz Viera $^{3}$, Ana Echavarria Velez ${ }^{4}$, Verónica Bravo Bravo ${ }^{1}$

${ }^{1}$ Universidad Técnica de Machala, Ecuador ${ }^{2}$ Instituto de Investigaciones para la Industria alimenticia, Cuba ${ }^{3}$ Universidad Tecnológica de La Habana "José Antonio Echeverría”, Cuba

${ }^{4}$ Universidad Estatal de Milagro, Ecuador *cnmatute@utmachala.edu.ec

\section{RESUMEN}

Se ha diseñado, evaluado y optimizado un jugo de tomate potencialmente funcional de alto valor biológico usando la cúrcuma (Cúrcuma longa) como agente antioxidante. La formulación del producto seleccionado se preparó en una escala piloto. A partir de las valoraciones sensoriales y análisis fisicoquímicos se ha obtenido la formulación óptima del concentrado de tomate (18 a $20^{\circ}$ Brix) 7,05 a $8,03 \%$, azúcar refinado $2,5 \%$, sal 0,8\%, 0,088\% de goma xantana, cúrcuma en polvo deshidratado (7\% de humedad) y 0.066 de agua. El contenido en fenol del producto envasado en vidrio fue de 171,4 $\mathrm{mg}$ de ácido gálico/L y la capacidad antioxidante fue de 1187,0 $\mu \mathrm{M} \mathrm{Fe2}$.

Palabras clave: Cúrcuma longa; alimento funcional; jugo de tomate; antioxidante.

\begin{abstract}
A potentially functional tomato juice of high biological value using turmeric (Curcuma longa) as an antioxidant agent has been designed, evaluated and optimized. The formula of the selected product was prepared on a pilot scale. Based on the sensory analysis and physicochemical tests, the optimum formula with tomato concentrate was found; (18-20 ${ }^{\circ}$ Brix) 7.5 to $8.3 \%$, refined sugar $2.5 \%$, salt $0.8 \%$, xanthan gum $0.088 \%$, dehydrated turmeric powder ( $7 \%$ humidity) and 0.066 water. The phenol content of the glass packaged product was of $171.4 \mathrm{mg}$ of gallic acid/L, and antioxidant capacity was of 1187,0 $\mu \mathrm{M} \mathrm{Fe} 2^{+}$.
\end{abstract}

Keywords: Turmeric (Curcuma longa); tomato juice; functional foods; antioxidants. 


\section{INTRODUCCIÓN}

El consumo de alimentos saludables ha sido una tendencia mundial en los últimos años, en gran parte debido al rápido crecimiento de enfermedades principalmente degenerativas y su estrecha relación con la alimentación, razón por la cual, se han generado en el mercado alimentos funcionales $(A F)$, enfocados a brindar al consumidor beneficios adicionales.

Según el Consejo de Alimentación y Nutrición de la Academia de Ciencias de los Estados Unidos, los $A F$ se definen como alimentos modificados o que contienen ingredientes que demuestren acciones que incrementan el bienestar del individuo o disminuyen los riesgos de padecer ciertas enfermedades, como resultado de sus ingredientes (prebióticos, probióticos, antioxidantes, ácidos grasos omega-3, ácido fólico, fitoesteroles, fitoestrógenos, etc.), o por haber removido aquellos componentes del alimento que pueden tener un efecto perjudicial en la salud.

Para Valenzuela, Sanhueza y Morales (2014), el consumo constante de sustancias antioxidantes se ha asociado a la prevención de la oxidación de los radicales libres producidos en la mayor parte de las células corporales como subproducto del metabolismo, evitando daño y destrucción celular.

La Curcuma longa L. (turmeric) ha evidenciado poseer un valor medicinal al descubrirse las propiedades antioxidantes de sus compuestos fenólicos, que son los denominados curcuminoides, aislándose tres curcuminoides principales, curcumina, dimetoxicurcumina y bidimetoxicurcumina. Todos ellos responsables del color dorado de las raíces de cúrcuma (Alvis, Arrazola y Martínez, 2012).

El tomate (Lycopersicum esculentum) contiene cantidades importantes de compuestos carotenoides como el Licopeno, además de vitamina $\mathrm{C}$, que de acuerdo con las investigaciones realizadas, demuestran tener actividad antioxidante (Laffita y Castillo, 2011).

Debido a las propiedades de la cúrcuma y al contenido importante de carotenoides que reportan los estudios realizados en tomate, la presen- te investigación se traza como objetivo general, diseñar un jugo de tomate potencialmente funcional con adición de cúrcuma como agente antioxidante, sin descuidar las características sensoriales que hagan del mismo un alimento de alta calidad nutricional, sensorialmente aceptado.

\section{MATERIALES Y MÉTODOS}

Materias primas: concentrado de tomate (18 a 20 \% sólidos solubles), cúrcuma de especie longa Linn seca en polvo con tamaño promedio de partícula (dp) 0,15 mm. La goma xantana (E 415) con 6-8 \% de humedad máxima y pureza de 91-108\%.

Caracterización de materias primas: a las muestras de concentrado de tomate se les realizó las siguientes determinaciones: humedad, sólidos solubles, azúcares reductores totales, acidez valorable, (expresado como porcentaje de ácido cítrico) $\mathrm{pH}$, contenido de ácido y carotenoides totales. El polvo de cúrcuma: humedad, hidratos de carbono, cenizas, almidón, proteínas (tomando 6,25 como factor de conversión del nitrógeno total presente en la muestra), extracto etéreo, ácido ascórbico, contenido de fenoles totales (Slinkard y Singlenton, 1977) y capacidad antioxidante (Benzie y Strain, 1996). Los hidratos de carbono se determinaron por diferencia. Todos los análisis se realizaron por triplicado, teniendo en cuenta los métodos oficiales regidos por las normas de alimentos (AOAC internacional).

Formulación del jugo de tomate con cúrcuma: para la formulación del jugo de tomate con cúrcuma, se tomó como base la producida en forma comercial, incluyéndose en esta la cúrcuma y como estabilizante la goma xantana. De esta forma se utilizaron los siguientes ingredientes: concentrado de tomate (18 - 20 \% s.s), azúcar refino, sal, ácido cítrico, agua, goma xantana y polvo de Cúrcuma longa Linn.

Establecimiento de los niveles de goma xantana y polvo de cúrcuma: el nivel de adición de cúrcuma se determinó partiendo de una concentración inicial máxima de $0,7 \%$, dosis diaria recomendada en la literatura por instituciones de salud (Jurenka, 2009), reduciendo paulatinamen- 
te hasta llegar a la dosis máxima admitida sensorialmente $0,125 \%$, evaluando como dosis mínima 0,025\%, ésta última prácticamente imperceptible al paladar.

Con el fin de determinar las cantidades de goma xantana necesarias para mantener en suspensión el polvo de cúrcuma, se realizaron pruebas de observación en un sistema modelo (agua destilada, cúrcuma 0,125\% y goma xantana), partiendo de un valor inicial de $0,2 \%$ de goma y evaluando visualmente la presencia de partículas en suspensión a las $24 \mathrm{~h}$ de preparada la muestra.

De esta forma se obtuvo que a concentraciones entre 0,05 y 0,15 \% (goma), se lograba una suspensión adecuada de las partículas. Una vez definidas las concentraciones de cúrcuma y goma a utilizar se procedió a realizar un diseño de superficie de respuesta D- óptimo utilizando el paquete estadístico Design - Expert (DX) versión 7.1.6 (Stat Ease2008). La matriz de este diseño se puede observar en la Tabla 1.

Tabla 1. Matriz del diseño de superficie de respuesta D- óptimo del Jugo de Tomate con Cúrcuma

\begin{tabular}{|lll|}
$\begin{array}{l}\text { NÚMERO DE CORRIDA } \\
\text { DE MUESTRA }\end{array}$ & $\begin{array}{l}\text { FACTOR 1: NIVEL } \\
\text { DE CURCUMA LON- } \\
\text { GA L (\%) }\end{array}$ & $\begin{array}{l}\text { FACTOR 2: NIVEL } \\
\text { DE GOMA (\%) }\end{array}$ \\
\hline 1 & 0,025 & 0,15 \\
\hline 2 & 0,025 & 0,05 \\
\hline 3 & 0,125 & 0,15 \\
\hline 4 & 0,125 & 0,05 \\
\hline 5 & 0,025 & 0,1 \\
\hline 6 & 0,125 & 0,15 \\
\hline 7 & 0,075 & 0,15 \\
\hline 8 & 0,075 & 0,075 \\
\hline 9 & 0,025 & 0,05 \\
\hline 10 & 0,125 & 0,05 \\
\hline 11 & 0,125 & 0,1 \\
\hline 12 & 0,05 & 0,125 \\
\hline Fuente: elaboración propia. & \\
\hline
\end{tabular}

Todas las corridas experimentales se realizaron a escala de laboratorio y se consideraron como variables respuesta del diseño, la viscosidad determinada en un viscosímetro Brokfield modelo LTV sp2 a $60 \mathrm{rpm}$ y temperatura de 25 $\left.{ }^{\circ} \mathrm{C}\right)$, y la respuesta sensorial evaluada por un equipo integrado por siete jueces entrenados, evaluándose los siguientes aspectos: separación de fases, cuerpo y amargor.

\section{Procesamiento estadístico}

Para analizar los resultados de las variables respuesta y establecer la formulación de la bebida, se aplicó la metodología de superficie de respuesta y se optimizaron las concentraciones de cúrcuma y goma, mediante el método numérico, poniendo restricciones en las variables, respuesta estadísticamente significativa, utilizándose el programa DX versión 7.1.6.

\section{Preparación del jugo}

Con la fórmula seleccionada se elaboró el jugo de tomate con cúrcuma a escala piloto siguiendo el flujo tecnológico que se describe a continuación: el proceso se inicia con el pesaje de las materias primas, con el fin de facilitar la dispersión se mezclan los ingredientes secos y se mezclan con el concentrado de tomate previamente disuelto en el agua. En un tanque de doble camisa de vapor se homogeniza y calienta la mezcla hasta llegar a ebullición, manteniéndola así durante cinco minutos agitando continuamente. Se envasa en caliente $\left(94-96^{\circ} \mathrm{C}\right)$ y pasteriza en baño maría durante cinco minutos; finalmente se enfría y almacena (Figura 1).

\section{RESULTADOS Y DISCUSIÓN}

Caracterización del concentrado de tomate y Cúrcuma longa Linn: los resultados de la caracterización físico - química y nutricional del concentrado de tomate se muestra en la tabla 2 .

Como se observa los índices de sólidos solubles, acidez y $\mathrm{pH}$, se encuentran en los intervalos de: sólidos solubles entre 18 y $20 \%$, acidez, entre 1,1 y $2,6 \%$ y pH inferior a 4,6. Por su parte las concentraciones de azúcares reductores, ácido ascórbico y carotenoides totales están en el entorno de lo señalado en la bibliografía consultada para este tipo de alimentos, en la que se encontraron valores para los azúcares reductores entre 


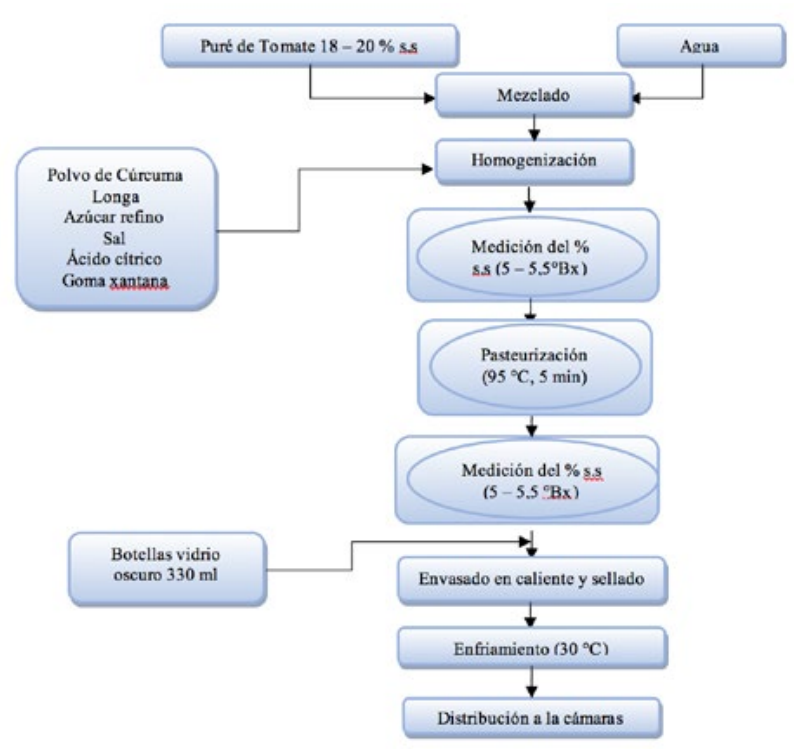

Figura 1. Diagrama de flujo del proceso de elaboración del jugo de tomate con cúrcuma.

Fuente: elaboración propia.

Tabla 2. Características físico - químicas y nutricionales del concentrado de tomate.

\begin{tabular}{|c|c|c|}
\hline & $\mathbf{x}$ & $\mathbf{S}$ \\
\hline Humedad (\%) & 82,75 & 0,35 \\
\hline Sólidos solubles ( $\left.{ }^{\circ} \mathrm{Brix}\right)$ & 18,25 & 0,35 \\
\hline $\mathrm{pH}$ & 4,22 & 0,02 \\
\hline Acidez valorable (\% de ácido cítrico) & 1,14 & 0,05 \\
\hline Azúcares reductores totales (\%) & 9,51 & 0,14 \\
\hline $\begin{array}{l}\text { Contenido de ácido ascórbico - Vitamina C } \\
(\mathrm{mg} / 100 \mathrm{~g})\end{array}$ & 21,95 & 0,74 \\
\hline Carotenoides Totales $(\mu \mathrm{g} / 100 \mathrm{~g})$ & 18707,5 & 79,9 \\
\hline
\end{tabular}

Fuente: elaboración propia.

Tabla 3. Características físico - químicas y nutricionales del polvo de cúrcuma longa Linn.

\begin{tabular}{|lll|}
\hline & $\mathbf{X}$ & $\mathrm{s}$ \\
\hline Humedad (\%) & 7,14 & 0,76 \\
\hline Cenizas (\%) & 8,27 & 0,10 \\
\hline Proteínas (\%) & 7,05 & 0,09 \\
\hline Hidratos de carbono totales & 76,01 & 0,49 \\
\hline Ácido ascórbico (mg/100 g) & 9,60 & 0.14 \\
\hline Almidón (\%) & 56,00 & 2,83 \\
\hline $\begin{array}{l}\text { Extracto etéreo (lípidos) } \\
\text { Fenoles totales (mg de ácido gá- }\end{array}$ & 3830 & 0,03 \\
\hline lico/100 g) & & 127,28 \\
\hline $\begin{array}{l}\text { Capacidad antioxidante ( } \mu \text { moles } \\
\text { Fe2+/ } 100 \mathrm{~g}\end{array}$ & 35950 & 1060 \\
\hline
\end{tabular}

Fuente: elaboración propia.
8 y 10 \%, ácido ascórbico entre 18 y 25 mg/100 g y contenido de carotenoides totales mayor a 1500 $\mu \mathrm{g} / 100 \mathrm{~g}$. A partir de esta caracterización, se puede decir que el concentrado de tomate cumple las especificaciones de calidad pertinentes (CONESA, 2011).

En la tabla 3 se muestran los resultados de la caracterización físico - química y nutricional del polvo de Cúrcuma longa Linn. Los valores obtenidos fueron comparables con los que reporta la literatura, apreciándose que el polvo obtenido de la cúrcuma posee características físico-químicas y nutricionales similares a las especies analizadas y reportados en la bibliografía consultada (Collison, 2014).

Formulación del jugo de tomate con cúrcuma: el efecto de la adición de la cúrcuma y goma xantana sobre los atributos sensoriales más importantes del jugo, se muestran a continuación.

Análisis de la superficie de respuesta para la separación de fases: tomando en consideración los coeficientes significativos del modelo codificado $(p<0,05)$ se obtuvo la ecuación 1 :

Separación de fases $=2,79-0,95 B \quad R^{2}=0,9063$ (Ec. 1).

Según el modelo obtenido, resultó significativo el contenido de G. xantana (factor B) ya que a medida que este aumenta en la formulación, la separación de fases disminuye, (figura 2) lo que es característico de este estabilizante. El contenido de cúrcuma en el jugo no presenta influencia significativa en la respuesta de la variable estudiada.

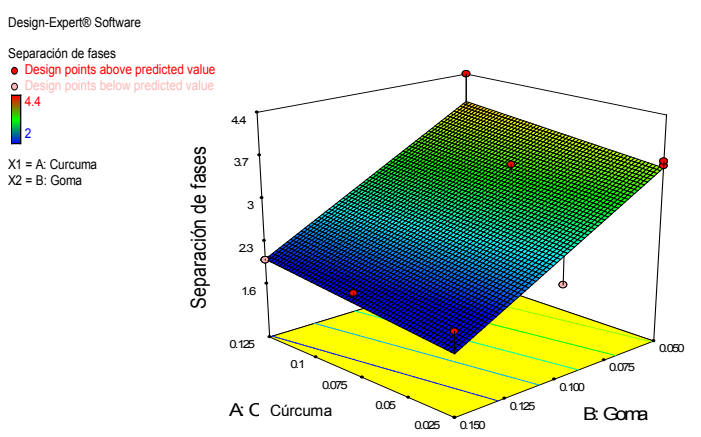

Figura 2. Superficie de respuesta para la separación de fases del jugo de tomate con cúrcuma Fuente: elaboración propia. 
Análisis de la superficie de respuesta para el cuerpo: tomando en cuenta los coeficientes significativos del modelo codificado $(p<0,05)$ se obtuvo la siguiente ecuación (2):

Cuerpo $=4,67+1,13 \mathrm{~B}-0,27 \mathrm{AB}-0,58 \mathrm{~B}^{2} \quad \mathrm{R}^{2}=0,9796$ (Ec.2).

A medida que se incrementa el nivel de goma, el cuerpo del jugo aumenta por su dependencia lineal positiva. Esta influencia disminuye en la medida que el nivel de goma se eleva. La dependencia del cuerpo con el término cuadrático de $\mathrm{B}$ indica no solo el incremento de dicho atributo con el aumento de la concentración de goma, sino que la dinámica de este aumento varía con la concentración del estabilizante.

Esta disminución de cuerpo se ve potenciada por la presencia del contenido de cúrcuma a consecuencia de la interacción negativa de los factores $\mathrm{AB}$. Efecto observado en la figura 3.

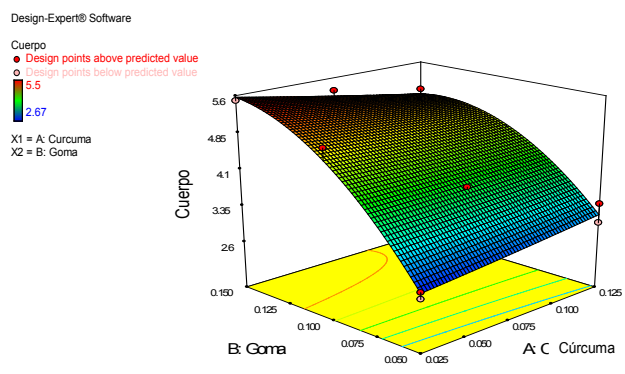

Figura 3. Superficie de respuesta para el cuerpo del jugo de tomate con cúrcuma

Fuente: elaboración propia.

\section{Análisis de la superficie de respuesta para el amargor}

Considerando los coeficientes significativos del modelo codificado $(p<0,05)$ se obtuvo la siguiente ecuación 3.

$$
\text { Amargor }=1,84+1,15 \mathrm{~A}+0,48 \mathrm{AB} \quad \mathrm{R}^{2}=0,8556
$$$$
\text { (Ec.3). }
$$

Se observa en el modelo que la cúrcuma (Factor A) y su interacción con la goma xantana (Factor B) influyen significativamente en la pun- tuación del amargor $(\mathrm{p}<0,05)$. Según se explica, existe una dependencia lineal positiva entre el contenido de cúrcuma y el amargor del jugo, por lo que a concentraciones más altas del rizoma se produce un aumento del amargor. Este efecto se ve incrementado con la elevación del nivel de goma xantana por la interacción de los factores, lo que se observa claramente en la superficie de respuesta obtenida (figura 4).

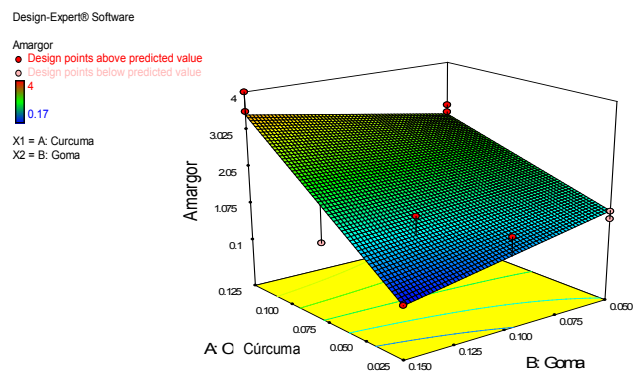

Figura 4. Superficie de respuesta para el amargor del jugo de tomate con cúrcuma Fuente: elaboración propia.

Efecto de la adición de la cúrcuma y goma xantana sobre la viscosidad: considerando los coeficientes significativos del modelo codificado $(p<0,05)$ se obtuvo la ecuación 5 .

Viscosidad $=30,85+8,57 \mathrm{~B} \quad \mathrm{R}^{2}=0,9484 \quad$ (Ec.5).

El modelo muestra que el único término significativo es la concentración de goma xantana (Factor B) teniendo una influencia lineal positiva en la viscosidad del jugo, incrementándose la respuesta a medida que se eleva el nivel de estabilizante en la formulación (figura 5).

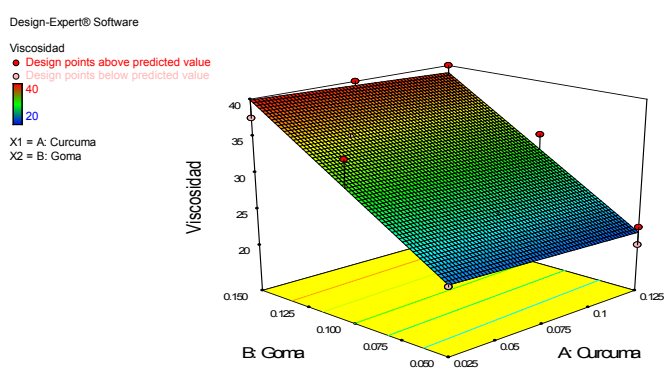

Figura 5. Superficie de respuesta para la viscosidad del jugo de tomate con cúrcuma Fuente: elaboración propia. 
Definición de la formulación del jugo de tomate con cúrcuma: tomando en consideración los resultados alcanzados en la modelación desarrollada para el efecto de la cúrcuma y goma xantana sobre los atributos sensoriales, se procedió a realizar una optimización numérica estableciendo restricciones a las variables respuesta (tabla 4). Los valores de las restricciones se seleccionaron en base a las características sensoriales esperadas en el producto final.

\begin{tabular}{|c|c|c|c|}
\hline \multirow[t]{2}{*}{ Variable respuesta } & \multicolumn{3}{|c|}{ Restricción } \\
\hline & $\begin{array}{l}\text { Límite } \\
\text { mínimo }\end{array}$ & $\begin{array}{l}\text { Límite } \\
\text { máximo }\end{array}$ & $\begin{array}{l}\text { Equivalencia } \\
\text { sensorial }\end{array}$ \\
\hline Separación de fases & 2 & 3 & $\begin{array}{l}\text { De muy ligero } \\
\text { a ligero }\end{array}$ \\
\hline Olor equilibrado & 4,5 & 5,5 & Equilibrado \\
\hline Sabor equilibrado & 4,5 & 5,5 & Equilibrado \\
\hline Amargor & 0 & 2 & Muy ligero \\
\hline $\begin{array}{l}\text { Equilibrio ácido - } \\
\text { dulce }\end{array}$ & 6,5 & 7,3 & Equilibrado \\
\hline Cuerpo & 2 & 4,5 & $\begin{array}{l}\text { De muy ligero } \\
\text { a ligero }\end{array}$ \\
\hline
\end{tabular}

Fuente: elaboración propia.

Considerando como meta principal, maximizar el contenido de cúrcuma y minimizar el contenido de goma, valorando la respuesta sensorial, se seleccionó la variante que se encuentran dentro de la zona óptima. De esta forma el jugo queda definido tal como muestra la tabla 5.

Tabla 5. Formulación del jugo de tomate con cúrcuma

\begin{tabular}{|c|c|}
\hline INGREDIENTE & $\begin{array}{l}\text { PORCENTAJE EN FOR- } \\
\text { MULA (\%) }\end{array}$ \\
\hline Agua & $88,06-88,9$ \\
\hline $\begin{array}{l}\text { Concentrado de tomate }(18-20 \\
\% \text { sólidos solubles) }\end{array}$ & $8,3-7,5$ \\
\hline Azúcar refino & 2,5 \\
\hline Sal & 0,8 \\
\hline Ácido cítrico & 0,15 \\
\hline Goma xantana & 0,088 \\
\hline Cúrcuma & 0,066 \\
\hline
\end{tabular}

Caracterización del jugo de tomate con cúrcuma obtenido a nivel piloto: según la caracteriza- ción físico - químicas y nutricionales del jugo de tomate con cúrcuma obtenido a nivel piloto (tabla 6) se infiere que el producto cumple con las especificaciones de calidad. Para el jugo de tomate se acepta un valor mínimo de 5\% de sólidos solubles y $1 \%$ como máximo en contenido de cloruros.

El contenido de ácido ascórbico en el jugo de tomate con cúrcuma resultó bajo. Esto pudo deberse a su baja concentración en el puré de tomate utilizado, o incluso a la posible pérdida de ácido ascórbico en el tratamiento térmico recibido. Por lo tanto el producto no puede considerarse fuente de esta vitamina.

Tabla 6. Caracterización del jugo de tomate con cúrcuma.

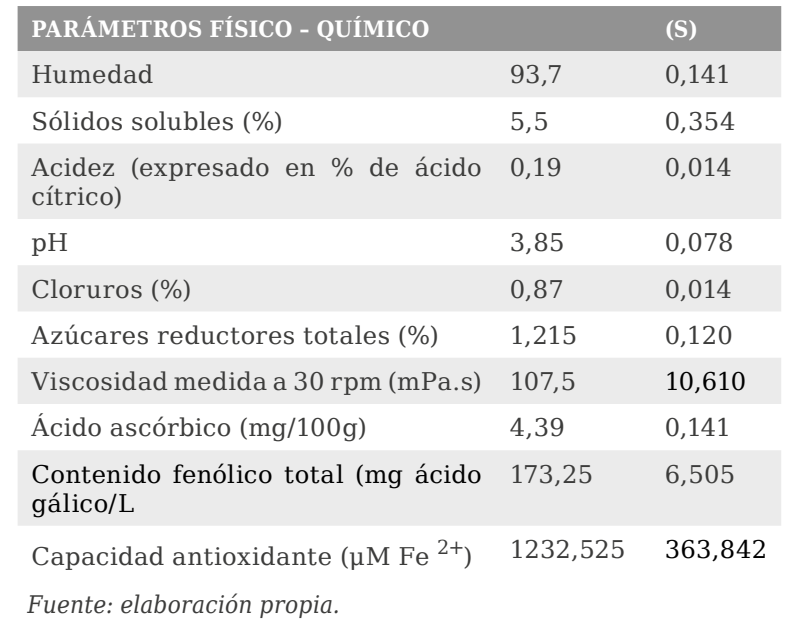

La formulación del jugo de tomate queda definida de la siguiente manera: concentrado de tomate (18 - $20 \%$ sólidos solubles) 7,5 - 8,3\%, azúcar refino $(2,5 \%)$, sal $(0,8 \%)$, goma xantana (0,088\%), polvo de Curcuma longa Linn (humedad 7 \% y (dp) de 0,15 mm) 0,066\% y agua (88 - 89\%).

El contenido de fenoles del jugo de tomate con cúrcuma envasado en botella de vidrio es de 171,4 mg de ácido gálico/L y la capacidad antioxidante del mismo es de 1187,0 $\mu \mathrm{M} \mathrm{Fe}{ }^{2+}$. Con ésta formulación se logró maximizar el contenido de cúrcuma y minimizar el contenido de goma, sin afectar la respuesta sensorial positiva de los panelistas ante el producto.

\section{Cumbres}




\section{REFERENCIAS BIBLIOGRÁFICAS}

Alvis, A.; Arrazola, G. y Martínez W. (2012). Evaluación de la Actividad y el Potencial Antioxidante de Extractos Hidro-Alcohólicos de Cúrcuma (Cúrcuma longa). Revista Información Tecnológica, 23(2):11-18

AOAC. (1995). Official Methods of Analysis of the Association of Official Analitycal Chemist. Arlington: Helrich K (Ed).

Benzie, I. y Strain, J. (1996). The ferric reducing ability of plasma (FRAP) as a measure of antioxidant power. The FRAP Assay Analitical Biochemestry, 23(9): 70 -76.

Collison, S. (2014). Curcumin and Inflammatory Diseases: Learn About Its Potential Role in Prevention and Treatment. Today's Dietitian, 16 (9): 56.

Conesa (2011). Especificaciones Técnicas para la fibra de tomate. Recuperado de: http:// www.e-conesa.com/docs/files/52enblanco.pdf

Donnelly, P.; Churilla, T.; Vinson, J. (2011). Análisis de la Capacidad Antioxidante y Antioxidantes/Caloría de Aguas Enriquecidas con Vitaminas y Bebidas Ricas en Polifenoles. México: Panamericana.

Echavarría, A.; Pagán, J y Ibarz, A. (2012). Melanoidins Formed by Maillard Reaction in Food and Their Biological Activity. Food Engineering Reviews, 4(4): 203-223

Jurenka, J. (2009). Anti-inflammatory properties of curcumin, a major constituent of Curcuma longa. Alternative Medicine Review, 14(2):141-53.

Laffita, C. y Castillo, A. (2011). Avances en la caracterización farmacotoxicológica de la planta medicinal Cúrcuma longa Linn. Revista MEDISAN, 16(1):97

Martínez, E. (2015). Caracterización morfológica y contenido de almidón resistente disponible en bananos (Musa sapientum) exportables del Ecuador. Revista Española de Nutrición Humana y Dietética, 19(3): 154.

Slinkard, K.; Singlenton, V. (1977). Total phenol analysis automation and comparison with manual method. American Journal Enology Viticulture. 28: 48-55.

Valenzuela, A.; Valenzuela, R.; Sanhueza, J.; Morales, G. (2014). Alimentos funcionales, nu- traceúticos y foshu: ¿vamos hacia un nuevo concepto de alimentación? Revista Chilena de Nutrición, 41(2).

Zamora, J. (2007). Micronutrientes en lucha por la salud. Revista Chilena de Nutrición, 34(1):17-26 